\title{
The role of Neopterin in cardiovascular disease
}

\section{II ruolo della neopterina nelle malattie cardiovascolari}

\author{
Mario Pacileo, Plinio Cirillo, Salvatore De Rosa, Grazia Ucci, Gianluca Petrillo, \\ Sergio Musto D’Amore, Laura Sasso, Paola Maietta, Raffaele Spagnuolo, Massimo Chiariello
}

\begin{abstract}
The role of Neopterin in cardiovascular disease. M. Pacileo, P. Cirillo, S. De Rosa, G. Ucci, G. Petrillo, S. Musto D’Amore, L. Sasso, P. Maietta, R. Spagnuolo, M. Chiariello. Inflammation plays a key role in the initiation and progression of atherosclerosis but also in the pathophysiology of atheromatous plaque disruption and the development of acute coronary syndromes. Neopterin is a marker of inflammation and of immune system activation, it is synthesized by macrophages, that, once activated, release this substance. Indeed, in clinical evaluation of patients, measurements of plasma levels of neopterin are usually used to evaluate progression of viral infections, renal transplant rejection, severe systemic inflammatory diseases, nephritic syndrome and several autoimmune diseases. This mediator is able to induce a pro-atherothrombotic phenotype in cells of the coronary circulation. Recent data indicate that serum levels of neopterin are elevated in patients with coronary and peripheral artery disease and seem to be a
\end{abstract}

prognostic marker for major adverse cardiovascular events. In particular, neopterin levels predict future major cardiac and vascular adverse events in patients presenting with chronic coronary artery disease, with acute coronary syndromes, and in those with critical limb ischemia. This renders this molecule a useful marker of atherosclerotic plaque activity, permitting the identification of the subjects at highest risk for major adverse cardiovascular events. In line with the above mentioned evidences, patients with high neopterin levels may require aggressive risk factor modification and intensive medical treatment irrespective of the severity of their coronary artery disease. This data suggest a potential clinical use of neopterin as a marker for disease activity in patients with cardiovascular disease.

Keywords: inflammation, neopterin, cardiovascular disease.

Monaldi Arch Chest Dis 2007; 68: 68-73.

Division of Cardiology, University of Naples “Federico II", Naples.

Corresponding author: Plinio Cirillo, MD, PhD; Division of Cardiology; University of Naples "Federico II"; Via Sergio Pansini 5 - I-80131 Naples, Italy; E-mail address: pcirillo@ unina.it

In the last two decades, systematic studies have provided convincing evidence that inflammation plays a key role both in the initiation and progression of atherosclerosis [1, 2]. In particular, it has been evidenced that several well-known markers of systemic inflammation such as C-reactive protein (CRP), amyloid A, interleukin-6, matrix metalloprotease(s) and others, have an important role in triggering atherosclerotic plaque formation as well as its grown. Indeed, these molecules might also actively participate in plaque destabilization, finally leading to the occurrence of acute coronary syndromes $[1,2]$.

Among these markers of inflammation, many studies have been focused on CRP. This inflammatory protein has been associated with increased cardiovascular risk in prospective epidemiological studies and it has been indicated as an important predictor of future cardiovascular adverse events [3]. In particular, patients with acute coronary syndromes and with elevated plasma levels of CRP have a significantly worse outcome as compared to patients with the same clinical presentation but with normal plasma levels of this protein [4]. Again, in healthy subjects, elevated CRP levels appear to be associated with the increased risk of develop future cardiovascular events [5]. Starting from these clinical observations, many studies have been designed to investigate the possible mechanism(s) of action of CRP, to clarify how this acute phase protein might be linked to cardiovascular events. Thus, it has been demonstrated that CRP, besides being a marker of inflammation, might exert direct effects on cells of the vessel wall directly involved in the pathophysiology of atherosclerosis and thrombosis such as endothelial and smooth muscle cells [6].

In recent years, some epidemiological studies have indicated that plasma levels of another wellknown marker of inflammation, neopterin, were significantly elevated in patients with coronary artery disease (CAD) and with acute coronary syndromes [7-10].

Neopterin is a pteridine compound and a byproduct of the guanosine triphosphate-biopterin pathway [11]. It is synthesized by macrophages, that, once activated, release this substance. Since macrophage are actively involved in inflammatory mechanisms, neopterin is considered an index of macrophage activity thus representing an inflammatory marker [12]. Indeed, in clinical evaluation of patients, measurements of plasma levels of neopterin are usually used to evaluate progression of viral infections, renal transplant rejection, severe systemic inflammatory diseases, nephritic syndrome and several autoimmune diseases [13]. In the relatively recent point of view that ascribes to inflammation an important role in the pathophysiology of atherosclerosis, it is intriguing to consider for neopterin an active role within vulnerable plaque in 
the mechanism(s) responsible of acute coronary syndromes.

In the present report we give a brief overview of clinical, epidemiological evidences witnessing neopterin role in atherothrombotic disease, illustrating the possible pathophysiological mechanim(s) involved in the modulation of these phenomena.

\section{Neopterin and Coronary Artery Disease}

In recent years, some epidemiological studies have pointed out the association existing between increased plasma levels of neopterin, coronary artery disease and acute coronary syndromes [7-10].

It has been demonstrated by Zouridakis et al. that, in patients with stable angina, a close relationship exists between increased plasma neopterin concentrations and a faster progression of coronary atherosclerotic disease [10]. In another study performed in patients with similar clinical characteristics, Avanzas et al. demonstrated that elevated neopterin concentrations were an independent predictor of major adverse coronary events [14]. Again, in patients with history of hypertension, clinical symptoms of chest pain and without angiographic evidence of atherosclerotic disease, elevated plasma levels of neopterin seem to be associated with a higher incidence of cardiac adverse events over a 1year follow up [15].

Interestingly, also in women, which represent a patient population which benefit of the protective effects of estrogens, Garcia-Moll et al. showed that elevated plasma concentrations of neopterin were associated with the significant increase of cardiovascular risk [16].

Particularly interesting was the observation that patients with unstable angina had higher measurable plasma levels of neopterin as compared to patients with stable angina [8], suggesting that this molecule might play a role in plaque destabilization. In line with this hypothesis, the same researchers evidenced, in patients with unstable angina which underwent to coronary angiography, that neopterin plasma levels were correlated with the number of complex lesions, but not with extent and severity of coronary atherosclerosis [17]. These data permit to speculate that neopterin might be a marker of "activity" of coronary disease rather than a measure of its extent.

To date, the possible correlation between plasma levels of neopterin and the extent of coronary atherosclerosis is still debated, because results of different research groups were quite different. Specifically, Tanaka et al. evidenced that higher neopterin concentrations were directly correlated to the severity of coronary atherosclerosis in patients with stable angina pectoris [18]. On the contrary, Schumacher et al. observed that increased neopterin levels in patients with stable CAD, were not associated to the number or extent of coronary vessel involvement. They failed to identify any significant difference in neopterin levels between patients with minor and severe CAD [8].

Neopterin levels are elevated also in patients with acute myocardial infarction. Gupta et al. showed that patients with unstable angina and myocardial infarc- tion have significantly higher neopterin levels than the patients of the control group or subjects with previous AMI [7].

As already described for CRP, several studies have demonstrated that neopterin might predict a worse outcome in patients with a first myocardial infarction. Specifically, Dominguez-Rodriguez et al. evidenced a strong association between neopterin levels and mortality in patients suffering acute myocardial infarction with ST-segment elevation [19]. Auer et al., in a retrospective study, observed that neopterin levels measured 72 hours after symptoms onset were significantly higher in patients with a clinical history of major cardiovascular events (MACE: cardiovascular death, recurrent AMI, stroke, hospital readmission for heart failure) during a $22 \pm 3$ months follow-up $[9,20]$. On the contrary, patients with the same clinical presentation at baseline, but with low neopterin levels measured at the same time-point, did not develop MACE. This predictive value of neopterin may be observed also in patients with non-Q-wave myocardial infarction, as demonstrated by Van Haelst et al. [21].

\section{Neopterin and peripheral artery disease}

Peripheral artery disease is usually associated with a higher frequency of concomitant coronary heart disease [22]. Thus, it is likely that neopterin levels are elevated also in patients with peripheral atherosclerosis. Indeed, Barani et al. evidenced that, in patients with critical limb ischemia (CLI), elevated levels of this inflammatory mediator are indicators of a more severe prognosis $[23,24]$. In particular, this study reported neopterin to be an independent risk variable for death within 1 year in patients with CLI [23]. Moreover, the same group evidenced that neopterin levels correlated positively with echocardiographic signs of congestive heart failure [24]. In addition, patients with ischemic ECG modifications presented higher neopterin concentrations and 1-year mortality rate [24].

According to the report by Barani, also Bertz et al. [25] reported that serum levels of neopterin, were elevated in patients with CLI. Specifically, these authors observed higher levels on neopterin in patients with CLI and diabetes as compared to patients with the same vascular disease but without diabetes. Moreover, they demonstrated a clear relationship between the extent of peripheral vascular disease, its severity and neopterin levels. In addition, to witness the correlation existing between peripheral and coronary artery disease, Erren et al. found higher neopterin levels in patients with peripheral arteries disease and concomitant coronary artery disease than in patients with coronary disease alone [26].

Also in patients with atherosclerotic disease of carotid artery, neopterin levels are usually elevated [27] and, interestingly, these elevated plasma concentrations are associated with an increased rate of stroke or transient ischemic attack [28].

\section{Neopterin and artery disease: pathophysiology}

Despite clinical observation described above that neopterin levels are strongly correlated to car- 
Table 1. - Neopterin and cardiovascular disease

\begin{tabular}{|c|c|c|}
\hline Author & Patients & Results \\
\hline Gupta et al. [7] & $\begin{array}{l}10 \text { pts with AMI } \\
67 \text { pts with remote AMI } \\
17 \text { pts with UA }\end{array}$ & $\begin{array}{l}\text { Pts with AMI and UA have significantly higher levels } \\
\text { of neopterin than pts with remote AMI or control-group } \\
\text { patients }\end{array}$ \\
\hline Schumacher et al. [8] & $\begin{array}{l}21 \text { pts with AMI } \\
62 \text { pts with stable CAD }\end{array}$ & $\begin{array}{l}\text { Neopterin was significantly increased in pts with stable CAD } \\
\text { and more pronounced in pts with AMI than control-group patients }\end{array}$ \\
\hline Auer et al. $[9,20]$ & $\begin{array}{l}25 \text { pts with ACS } \\
31 \text { pts with stable CAD }\end{array}$ & $\begin{array}{l}\text { Neopterin is significantly increased in pts with ACS and } \\
\text { elevated neopterin levels predict MACE during follow-up }\end{array}$ \\
\hline Zouridakis et al. [10] & 124 pts with stable CAD & $\begin{array}{l}\text { Rapid CAD progression in pts with stable CAD is associated } \\
\text { with increased neopterin levels }\end{array}$ \\
\hline Avanzas et al. [14] & 297 pts with stable CAD & $\begin{array}{l}\text { Serum neopterin levels is an independent predictor of MACE } \\
\text { in pts with stable CAD }\end{array}$ \\
\hline Avanzas et al. [15] & $\begin{array}{l}58 \text { pts with hypertension } \\
\text { and without obstructive } \\
\text { CAD }\end{array}$ & $\begin{array}{l}\text { Pts who developed MACE during follow-up had significantly } \\
\text { higher neopterin levels than control-group patients }\end{array}$ \\
\hline Garcia-Moll et al. [16] & $\begin{array}{l}82 \text { pts with stable CAD } \\
32 \text { pts with UA }\end{array}$ & $\begin{array}{l}\text { Pts with UA had significantly higher neopterin levels than pts } \\
\text { with stable CAD. Pts with stable CAD and with MACE during } \\
\text { follow-up had significantly higher neopterin levels than those } \\
\text { without MACE }\end{array}$ \\
\hline Garcia-Moll et al. [17] & $\begin{array}{l}82 \text { pts with stable CAD } \\
50 \text { pts with UA }\end{array}$ & $\begin{array}{l}\text { Neopterin levels is associated with the presence } \\
\text { of angiographically demonstrated complex lesions }\end{array}$ \\
\hline Tanaka et al. [18] & 78 pts with stable CAD & Neopterin levels is a useful marker of the severity CAD \\
\hline Dominguez-Rodriguez et al. [19] & 82 pts with AMI & Serum neopterin levels predict MACE in pts with AMI \\
\hline van Haelst et al. [21] & 210 pts with NQMI & Serum neopterin levels predict MACE in pts with NQMI \\
\hline Barani et al. [23] & 259 pts with CLI & $\begin{array}{l}\text { Serum neopterin levels is an independent predictor of 1-year } \\
\text { mortality in pts with CLI }\end{array}$ \\
\hline Barani et al. [24] & 232 pts with CLI & $\begin{array}{l}\text { Neopterin concentration correlated positively with echocardiographic } \\
\text { signs of congestive heart failure and it is an indicators of a more } \\
\text { severe prognosis in pts with CLI }\end{array}$ \\
\hline Bertz et al. [25] & 259 pts with CLI & $\begin{array}{l}\text { Neopterin is significantly increased in pts with CLI and diabetes } \\
\text { than pts with CLI without diabetes }\end{array}$ \\
\hline Erren et al. [26] & $\begin{array}{l}57 \text { pts with CAD } \\
17 \text { pts with CAD and PAD } \\
2 \text { pts with PAD }\end{array}$ & $\begin{array}{l}\text { Pts with CAD and PAD have higher neopterin levels than in pts } \\
\text { with CAD alone }\end{array}$ \\
\hline Weiss et al. [27] & $561 \mathrm{pts}$ & $\begin{array}{l}\text { Pts with carotid atherosclerosis have higher neopterin levels } \\
\text { than in pts without }\end{array}$ \\
\hline Anwaar et al. [28] & 59 pts with stroke & Plasma neopterin increased after stroke \\
\hline
\end{tabular}

AMI: acute myocardial infarction; UA: unstable angina; CAD: coronary artery disease; ACS: acute coronary syndromes; MACE: major cardiovascular adverse events; NQMI: non-Q myocardial infarction; CLI: critical limb ischemia; PAD: peripheral artery disease.

diovascular adverse events in patients with coronary as well as peripheral artery disease, the pathophysiology of these phenomena still remains largely incomplete. Indeed, as for CRP, the story goes from "bedside to the bench" because the researchers have focused their attention to the cardiovascular mechanisms of action of neopterin only in recent times.

Studies dealing with the potential effects of neopterin provided evidence that promotion of oxidative stress is a fundamental principle of neopter- in's mode of action [29]. In particular, it has been recently demonstrated that neopterin is able to determine oxidative stress by means of iNOS induction [30]. Oxygen free radicals are highly reactive molecules with well-known effects on cardiovascular system [31]. Thus, it is very likely that neopterin might exert its effects on vessel wall cells thought this mechanism of action.

In line with these previous observation, we have recently demonstrated that neopterin has direct ef- 


\section{Inflammation}

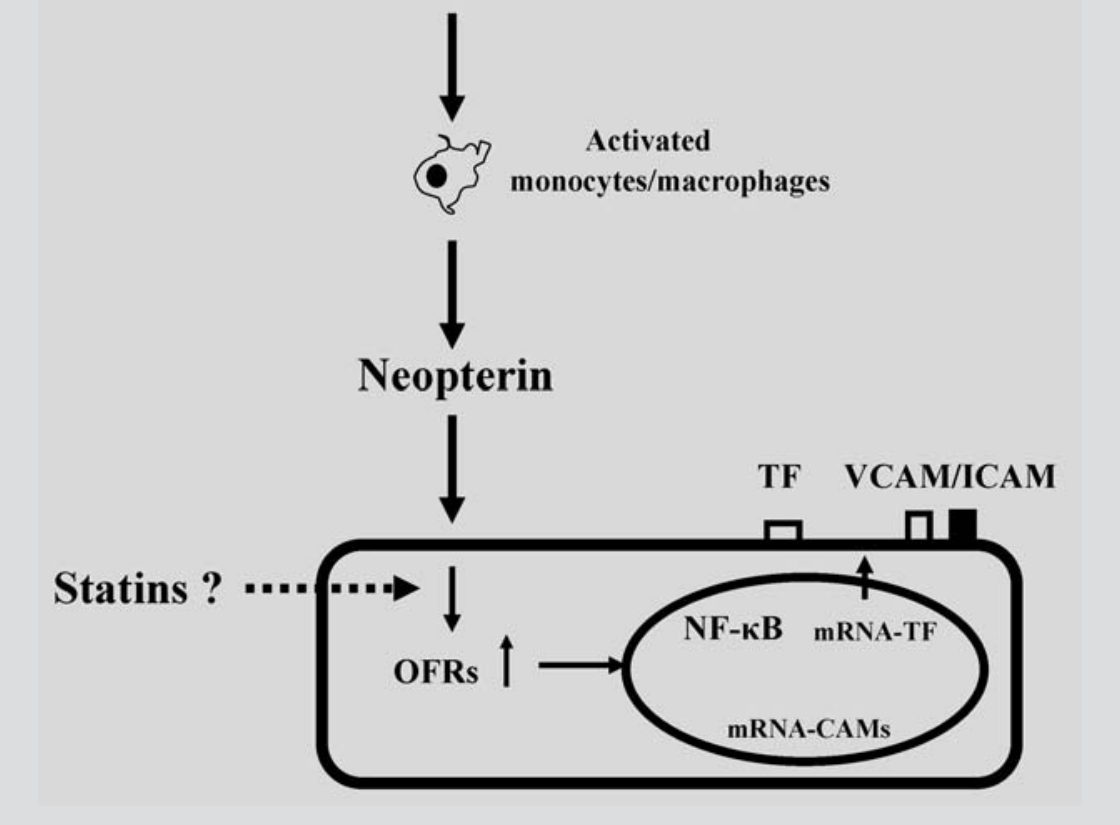

Figure 1. - The inflammation play a role key in the pathogenesis of atherosclerosis. Activated monocytes/macrophages in atherosclerotic lesions significantly increase local neopterin levels. Neopterin stimulates cytoplasmatic oxygen free radical generation. OFRs activate nuclear transcription factor NF- $\kappa \mathrm{B}$. This transcription factor is responsible of mRNA transcription for Tissue Factor and Cellular Adhesion Molecules. Statins might reduce these neopterin effects by inhibiting OFRs production. OFRs: oxygen free radicals; TF: tissue factor; CAMs: cellular adhesion molecules.

fects on human cells of the coronary circulation shifting them towards a pro-atherothrombotic phenotype [32]. Specifically, in our study we have evidenced that this molecule plays an active role in the initiation of the atherosclerotic disease by inducing, in endothelial cells, the expression of two important Cellular Adhesion Molecules (CAMs) such as VCAM-1 and ICAM-1. These CAMs are responsible of attachment and trans-endothelial migration of leukocytes [33] and are known to be directly involved in the pathogenesis of atherosclerosis mediating many of the stages of the disease progression [33]. In addition, serum concentration of CAMs are strongly correlated with coronary artery disease [34] and with the risk of cardiovascular events [35]. Furthermore, neopterin is able to induce expression on endothelial cells of Tissue Factor (TF), the initiator of the extrinsic coagulation pathway, and trigger of intravascular thrombosis [36, 37]. These observations might have important pathophysiological consequences, since endothelial cells represent the interface between the vessel wall and the flowing bloodstream containing coagulation factors and leukocytes. These neopterin effects on coronary endothelial cells were mediated through oxygen free radicals production and activation of the transcription factor NF- $\kappa \mathrm{B}$. This transcription factor is present but inactive in the cytoplasm of many cells such as lymphocytes, monocytes, endothelial and smooth muscle cells. It seems to be activated by several stimuli during the atherosclerotic process and it is responsible of the expression of inflammatory proteins that actively participate in this process and might lead to plaque disruption and acute coronary events [38]. In particular, activation of NF- $\kappa \mathrm{B}$ stim- ulates the promoter for TF [39], for CAMs [40] as well as for Nitric Oxide genes [41].

Considering that NF- $\mathrm{\kappa B}$ has been demonstrated to be activated in the peripheral monocytes [42], as well as within the unstable plaques of patients with acute coronary syndromes [43], the finding that neopterin induces $\mathrm{TF}$ and CAMs expression via activation of $\mathrm{NF}-\kappa \mathrm{B}$ might explain, at least in part, why patients with artery disease and elevated neopterin serum levels have a worse clinical outcome than patients with normal serum levels.

Taken together, these data permit to design an hypothetical mechanism of action in which activated monocytes/macrophages in atherosclerotic lesions significantly increase local neopterin levels; thus, they induce expression of ICAM-1 and VCAM-1 on endothelial cells in coronary circulation. Increased expression of CAMs may result in increase binding of T lymphocytes, platelets and other monocytes (that, once activated, further increase local neopterin concentration), key players in the atherosclerotic process. The endothelial activation causes expression of TF, promoting an "endothelial pro-thrombotic phenotype" and finally leading to the occurrence of acute coronary syndromes.

\section{Conclusions}

The studies reviewed have demonstrated that neopterin levels are an important predictor of future cardiac as well as vascular adverse events. In particular, neopterin levels predict future major cardiac and vascular adverse events in patients presenting with chronic coronary artery disease $[8,10,14,16]$, with acute coronary syndromes [7-9, 17, 19-21], and in those with CLI $[23,24]$. Many of the above cited studies underline the strong association between high neopterin levels and complex atherosclerotic lesions but not with disease extension [8, 14, 16, 17], giving account for the prognostic properties of neopterin. This renders this molecule a useful marker of atherosclerotic plaque activity, permitting the identification of the subjects at highest risk for MACE.

Several researchers have reported the beneficial effects of pharmacological intervention with statins in reducing cardiovascular disease risk [44, 45]. This evidence provides an adjunctive hint to the pathophysiology of plaque instability, suggesting that at least part of the benefit observed using these medications are related to their anti-inflammatory effects. Indeed, it has been demonstrated, in vitro, that neopterin effects on endothelial cells are significantly reduced by lovastatin [32]. In line with the 
above mentioned evidences, patients with high neopterin levels may require aggressive risk factor modification and intensive medical treatment irrespective of the severity of their coronary artery disease.

Taken together, observations from all the studies reviewed propose the existence of a strong link between high neopterin levels and cardiovascular risk profile, suggesting a potential clinical use of neopterin as a marker for disease activity in subject with cardiovascular disease. This could help in identifying patients who are at a higher risk of developing cardiovascular adverse events who might benefit from urgent preventive strategies exploitation or extensive diagnostic work-up, as well as actual therapy, depending from their co-morbidities.

\section{Riassunto}

L'infiammazione gioca un ruolo chiave nello sviluppo e nella progressione della patologia aterosclerotica, ma anche nella fisiopatologia della rottura della placca ateromasica e nello sviluppo delle sindromi coronariche acute. La neopterina è un marker infiammatorio oltre che di attivazione del sistema immunitario, sintetizzato dai macrofagi che una volta attivati rilasciano tale sostanza. Tale molecola è adoperata usualmente in clinica per la valutazione della progressione di infezioni virali, rigetto del trapianto renale, severe patologie infiammatorie sistemiche, sindromi nefritiche e patologie autoimmunitarie. La neopterina è capace di indurre un fenotipo pro-aterotrombotico in cellule del circolo coronarico. Recenti evidenze cliniche indicano che i livelli plasmatici di tale molecola sono incrementati in pazienti con patologia del circolo arterioso coronarico o periferico e tale marker ha dimostrato di avere un peso prognostico nello sviluppo di maggiori eventi avversi cardiovascolari. Tutto ciò determina che tale molecola risulta un utile marker di attività della placca aterosclerotica permettendo l'identificazione di soggetti a più alto rischio per future eventi avversi cardiovascolari, che richiedono un trattamento più aggressivo dei fattori di rischio cardiovascolari. Questo lavoro suggerisce un potenziale clinico utilizzo della neopterina come marker di rischio in pazienti con patologie cardiovascolari.

\section{LIST OF ABBREVIATIONS}

CRP: C-reactive protein

IL-6: interleukin-6

MMPs: matrix metalloproteases

CAD: coronary artery disease

AMI: acute myocardial infarction

MACE: major adverse cardiovascular events

CLI: critical limb ischemia

ECG: electrocardiogram

CAMs: cellular adhesion molecules

TF: tissue factor

UA: unstable angina

ACS: acute coronary syndromes

NQMI: non-Q myocardial infarction

PAD: peripheral artery disease

\section{References}

1. Ross R. The pathogenesis of atherosclerosis: a perspective for the 1990s. Nature 1993; 362: 801-809.

2. Libby P, Ridker PM, Maseri A. Inflammation and atherosclerosis. Circulation 2002; 105: 801-809.

3. de Ferranti SD, Rifai N. C-reactive protein: a nontraditional serum marker of cardiovascular risk. Cardiovasc Pathol 2007; 16: 14-21.

4. Kavsak PA, Macrae AR, Newman AM, et al. Elevated Creactive protein in acute coronary syndrome presentation is an independent predictor of long-term mortality and heart failure. Clin Biochem 2007; 40: 326-9.

5. Ridker PM. High-sensitivity C-reactive protein and cardiovascular risk: rationale for screening and primary prevention. Am J Cardiol 2003; 92: 17K-22K.

6. Cirillo P, Golino P, Calabro P, et al. C-reactive protein induces tissue factor expression and promotes smooth muscle and endothelial cell proliferation. Cardiovasc Res 2005; 68: 47-55.

7. Gupta S, Fredericks S, Schwartzman RA, et al. Serum neopterin in acute coronary syndromes. Lancet 1997; 349: 1252-1253.

8. Schumacher M, Halwachs G, Tatzber F, et al. Increased Neopterin in patients with chronic and acute coronary syndromes. J Am Coll Cardiol 1997; 30: 703-707.

9. Auer J, Berent R, Lassnig E, Weber T, Eber B. Prognostic significance of immune activation after acute coronary syndromes. J Am Coll Cardiol 2002; 39: 1878.

10. Zouridakis E, Avanzas P, Arroyo-Espliguero R, Fredericks S, Kaski JC. Markers of inflammation and rapid coronary artery disease progression in patients with stable angina pectoris. Circulation 2004; 110: 1747-1753.

11. Werner-Felmayer G., Werner ER, Fuchs D, et al. Pteridine biosyntesis in human endotelial cells. Impact on nitric oxide-mediated formation of cyclic GMP. J Biol Chem 1993.

12. Huber C, Batchelor JR, Fuchs D, et al. Immune responseassociated production of neopterin. Release from macrophages primarily under control of interferon-gamma. J Exp Med 1984; 160: 310-316.

13. Fuchs D, Weiss G, Reibnegger G, Wachter H. The role of neopterin as a monitor of cellular immune activation in transplantation, inflammatory, infectious, and malignant diseases. Crit Rev Clin Lab Sci 1992; 29: 307-41.

14. Avanzas P, Arroyo-Espliguero R, Quiles J, Roy D, Kaski JC. Elevated serum neopterin predicts future adverse cardiac in patients with chronic stable angina pectoris. Eur Heart J 2005; 26: 457-463.

15. Avanzas P, Arroyo-Espliguero R, Cosin-Sales J, Quiles J, Zouridakis E, Kaski JC. Prognostic value of neopterin levels in treated patients with hypertension and chest pain but without obstructive coronary artery disease. Am J Cardiol 2004; 93: 627-629.

16. Garcia-Moll X, Cole D, Zouridakis E, Kaski JC. Increased serum neopterin: a marker of coronary artery disease activity in women. Heart 2000; 83: 346-350.

17. Garcia-Moll X, Coccolo F, Cole D, Kaski JC. Serum neopterin and complex stenosis morphology in patients with unstable angina. J Am Coll Cardiol 2000; 35: 956962.

18. Tanaka T, Nakamura Y, Nasuno A, et al. Plasma concentrations of monocyte chemoattractant protein 1 (MCP-1) and neopterin in the coronary circulation of patients with coronary artery disease. Circ J 2004; 68: 114-20.

19. Dominguez-Rodriguez A, Abreu-Gonzalez P, GarciaGonzalez M. Usefulness of neopterin levels and left ventricular function for risk assessment in survivors of acute myocardial infarction. Int J Cardiol 2006 10; 111: 318-20.

20. Auer J, Berent R, Lassnig E, Eber B. Serum neopterin and activity of coronary artery disease. Heart disease 2001; 3 : 297-301. 
21. van Haelst PL, Liem A, van Boven AJ, et al. Usefulness of elevated neopterin and C-reactive protein levels in predicting cardiovascular events in patients with non-Q-wave myocardial infarction. Am J Cardiol 2003; 92: 1201-3.

22. Aronow WS. Peripheral arterial disease. Geriatrics 2007; 62: 19-25.

23. Barani J, Nilsson JA, Mattiasson I, Lindblad B, Gottsater A. Inflammatory mediators are associated with 1-year mortality in critical limb ischemia. J Vasc Surg 2005; 42: 75-80.

24. Barani J, Mattiasson I, Lindblad B, Gottsäter A. Cardiac function, inflammatory mediators and mortality in critical limb ischemia. Angiology 2006; 57: 437-44.

25. Bertz L, Barani J, Gottsäter A, Nilsson PM, Mattiasson I, Lindblad B. Are there differences of inflammatory biomarkers between diabetic and non-diabetic patients with critical limb ischemia? Int Angiol 2006; 25: 370-7.

26. Erren M, Reinecke H, Junker R, et al. Systemic inflammatory parameters in patients with atherosclerosis of the coronary and peripheral arteries. Arterioscler Thromb Vasc Biol 1999; 19: 2355-63.

27. Weiss G, Willeit J, Kiechl S, et al. Increased concentrations of neopterin in carotid atherosclerosis. Atherosclerosis 1994; 106: 263-71.

28. Anwaar I, Gottsater A, Lindgarde F, Mattiasson I. Increasing plasma neopterin and persistent plasma endothelin during follow-up after acute cerebral ischemia. Angiology 1999; 50: 1-8.

29. Oettl K, Reibnegger G. Pteridine derivatives as modulators of oxidative stress. Curr Drug Metab 2002; 3: 203-9.

30. Schobersberger W, Hoffmann G, Grote J, Wachter H, Fuchs D. Induction of inducible nitric oxide synthase expression by neopterin in vascular smooth muscle cells. FEBS Lett 1995; 377: 461-4.

31. Giordano FJ. Oxygen, oxidative stress, hypoxia, and heart failure. J Clin Invest 2005; 115: 500-8.

32. Cirillo P, Pacileo M, De Rosa S, et al. Neopterin induces pro-atherothrombotic phenotype in human coronary endothelial cells. Thromb Haemost 2006; 4: 2248-2255.

33. Adams DH, Shaw S. Leucocyte-endothelial interactions and regulation of leucocyte migration. Lancet 1994; 343 : 831-6.

34. Morisaki N, Saito I, Tamura K, et al. New indices of ischemic heart disease and aging: studies on the serum levels of soluble intercellular adhesion molecule-1 (ICAM-1) and soluble vascular cell adhesion molecule-1 (VCAM-1) in patients with hypercholesterolemia and ischemic heart disease. Atherosclerosis 1997; 131: 43-8.

35. Ridker PM, Hennekens CH, Roitman-Johnson B, Stampfer MJ, Allen J. Plasma concentration of soluble intercellular adhesion molecule 1 and risks of future myocardial infarction in apparently healthy men. Lancet 1998; 351: 88-92.

36. Pawashe AB, Golino P, Ambrosio G, et al. A monoclonal antibody against rabbit tissue factor inhibits thrombus formation in stenotic injured rabbit carotid arteries. Circ Res 1994; 74: 56-63.

37. Ragni M, Cirillo P, Pascucci I, et al. Monoclonal antibody against tissue factor shortens tissue plasminogen activator lysis time and prevents reocclusion in a rabbit model of carotid artery thrombosis. Circulation 1996; 93 : 1913-8.

38. Barnes PJ, Karin M. Nuclear factor-kappaB: a pivotal transcription factor in chronic inflammatory diseases. $N$ Engl J Med 1997; 336: 1066-71.

39. Mackman N. Regulation of the tissue factor gene. Thromb Haemost. 1997; 78: 747-54.

40. Collins T, Read MA, Neish AS, Whitley MZ, Thanos D, Maniatis T. Transcriptional regulation of endothelial cell adhesion molecules: NF-kappa B and cytokine-inducible enhancers. FASEB J 1995; 9: 899-909.

41. Yan ZQ, Sirsjo A, Bochaton-Piallat ML, Gabbiani G, Hansson GK. Augmented expression of inducible NO synthase in vascular smooth muscle cells during aging is associated with enhanced NF-kappaB activation. Arterioscler Thromb Vasc Biol 1999; 19: 2854-62.

42. Ritchie ME. Nuclear factor-kappaB is selectively and markedly activated in humans with unstable angina pectoris. Circulation 1998; 98: 1707-13.

43. Wilson SH, Best PJ, Edwards WD, et al. Nuclear factorkappaB immunoreactivity is present in human coronary plaque and enhanced in patients with unstable angina pectoris. Atherosclerosis 2002; 160: 147-53.

44. Farmer JA, Gotto AM Jr. The Heart Protection Study: expanding the boundaries for high-risk coronary disease prevention. Am J Cardiol 2003; 92: 3i-9i.

45. Pedersen TR, Wilhelmsen L, Faergeman O, et al. Followup study of patients randomized in the Scandinavian simvastatin survival study (4S) of cholesterol lowering. Am J Cardiol 2000 Aug 1; 86 (3): 257-62. 